\title{
Automatic Column Assignment for Block World Domain with PNRD/iPNRD *
}

\author{
G.A. Souza ${ }^{1 *}$ H.B. Silva ${ }^{2 *}$ J.J.P.Z.S. Tavares ${ }^{3 *}$ \\ * Faculdade de Engenharia Mecânica, Universidade Federal de \\ Uberlândia, MG, (e-mail: gabrielsouzaworking@gmail.com ${ }^{1}$, \\ henriquebritos@hotmail.com ${ }^{2}$, jean.tavares@ufu.br $\left.{ }^{3}\right)$.
}

\begin{abstract}
Too much effort is being invested in automated planning field, however, this area has been focusing more on improving the performance of search algorithms in an abstract space than on practical solutions. Among several challenges it can be pointed out replanning, uncertanties and partial observation. This paper presents an automatic column assignment for an adaptive control of a robotic arm and three blocks based on PNRD - Petri Net inside RFID Database - and iPNRD - inverted PNRD - integration. The procedure is composed by building a Petri space, PNRD, and iPNRD model for each agent, and by integrating their information so that the robotic arm may autonomously order the blocks to the desired final global state, stored previously in each block's RFID tag; comparing the logical initial position with the physical position identification; assigning automatically the column index; generating a sequence of movements; and executing related actions. Some issues arise because of the dispersed information, physical-logical interface and its constraints, requiring the detection of not conformance in results, optimally solve and realize the required sorting given its partial observably, and sequential movement of one block each time. The feedback from PNRD/iPNRD models with physical positioning can certify the required initial, intermediate and final condition and also check for exceptions, resulting in an adaptive robotic control.
\end{abstract}

Keywords: Block Worlds, PNRD/iPNRD, Automatic Assignment, Adaptive Control.

\section{INTRODUCTION}

It is a well known fact that the AI planning community is very committed to apply the developments already achieved in this area to real complex applications. However realistic planning problems bring great challenges not only during design processes but also for the automated planners during the planning process itself (Sette et al., 2008). According to Ghallab, Nau and Traverso (2004) there are many reasons for the low adoption of automated planning in the industry. First, they rely on the widely used 'preconditions-and-effects' representations that provide predictive models of actions often too abstract to be of great use by actors. The automated planning field has been focusing more on improving the performance of search algorithms in an abstract space than on improving its models' predictive capabilities. Planning is easily formalized, whereas acting is not. There is a clear frontier between planning and performing, but the borderline between acting and performing is much more blurred. In addition, automated plans are usually an easy packaged input-output function, although actor's deliberation functions are difficult to integrate to open and dynamic environments. Such changes on focus entail two interconnected principles: a hierarchical structure to integrate the actor's deliberation function, and the continual online planning and reasoning throughout the acting process. Fonseca et

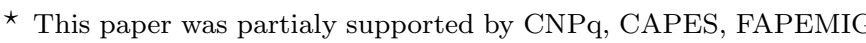
and UFU.
}

al. (2016) questioned when automatic planners would be integrated with industrial systems and presented a solution based on Grafcet. However, online process redesign and replanning is still an open issue. Partial observed systems are also a challenge.

Towards assisting the development of a practical replanning tool, this paper presents an automatic column assignment for an adaptive control of a robotic arm and three blocks based on PNRD - Petri Net inside RFID Database - and iPNRD - inverted PNRD - integration. A didactic example is presented with three block and one robotic arm. This paper is structured as follows, the next section presents preliminares with PNRD/iPNRD fundamentals and block worlds. Section 3 shows previous work; followed by the automatic column assignment and movement optimization. Some conclusions and further works are presented.

\section{PRELIMINARIES}

Problem and tool definitions are necessary to understand the sorting process and improvements. The Petri Net models are useful for full state representation and searching (Murata, 1989), while the Blocks World is a very famous and explored toy problem in AI research (Gupta, 1992).

\subsection{PNRD and $i P N R D$}

PNRD (Tavares and Saraiva, 2010) and iPNRD (Fonseca, 2018) are graphical and mathematical models based on PN 
PNRD

iPNRD

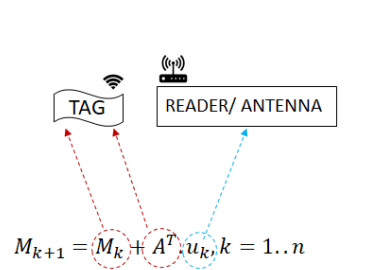

Datalog (userID - timestamp)

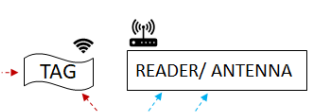

$M_{k+1}=M_{k}, A^{T}, u_{k}, k=1 . n$

$M_{k+1}=M_{k}+A^{\top}, u_{k}, k=1 . . n$

Fig. 1. PNRD and iPNRD Approach (Fonseca, 2018).

(Petri Nets) for representing discrete states and change mechanisms. They use RFID apparatus for data extraction and manipulation and the acquired database can be used for analysis. RFID is a technology used for digital data storage in smart labels. It does not require direct physical contact, direct line-of-sight and can store/retrieve more data than simple sensors. The basic components involved are tags and readers, used as labels and for identification/manipulation, respectively. Tags stores an unique identification data, and an exclusive identity in database. According to Murata (1989), PN are a conceptual graphical and mathematical model. PN provide the basic primitives for communication, concurrence and synchronization modeling and a graphical notation. Formally, an elementary $\mathrm{PN}$ is defined as the four tuple $P N=\left(P, T, F, M_{0}\right)$, in which $P$ is a finite set of places, $T$ is a finite set of transitions where $P \cap T=, F \subseteq(T \times P) \cap(P \times T)$ is the flow relation, and $M_{0}$ is the initial marking vector. PN can be represented mathematically as a matrix equation presented in equation (1), where $M_{k+1}$ is the next marking vector, $M_{k}$ is the current marking vector, $A^{t}$ is the incident matrix and $u_{k}$ is the firing vector.

$$
M_{k+1}=M_{k}+A^{t} \cdot u_{k}
$$

As defined in Tavares and Saraiva (2010) the PNRD approach applies PN as a formal data structure distributed across RFID components. The tag PNRD data can be defined by the object incidence matrix $A^{t}$ and its current state $M_{k}$. The RFID readers are linked to the firing vector $u_{k}$, so that they can perform equation (1) during tag data capture.

The iPNRD approach (Fonseca, 2018) inverts the RFID components storage, it means, tag stores the firing vector $u_{k}$ and additional data, while readers holds the incidence matrix $A^{t}$ and its current state $M_{k}$. Figure 1 shows a general PNRD and iPNRD schema.

\subsection{Blocks World}

The objective in Blocks World is to arrange the blocks from an initial configuration to a specified one with minimal cost respecting the constraints. It is one of the most famous planning domains in AI (Ghallab, Nau and Traverso, 2004). The Block Worlds rules can vary between distinct authors, but the most common rules are only one block can be moved at a time; a block can only be moved if there is no other block atop; each block has an unique identity.

The Blocks World explored here is a $3 \times 3$ discrete position grid, with three different blocks endowed with unique identity.The notation system is defined as if block $X$ is atop block $Y$, it can be represented by $X Y$. If the blocks are in different columns, the can be represented by $X_{-} Y$. For example $C B_{-} A$ means that block $C$ is over $B$ and both are beside $A$. $B$ and $A$ are implicitly atop the table. This notation is logically suitable, however, ambiguity problems arise because a logical representation can be materially disposed in many ways. $A_{-} B_{-} C$ can be disposed in 6 ways, depending on with column is each block. Usually researchers solve this ambiguity defining a specific column for each block when it is directly atop the table. This work presents a method to counteract this restriction.

Blocks World has been shown to be a difficult problem for finding an optimal plan, because it is categorized as NP-hard in the worst case (Gupta and Nau, 1992). The optimal path is defined as the global state route that has minimal cost, where cost is adopted as the total applied transitions, all having unitary cost.

\section{PREVIOUS WORKS}

As presented in Souza and Tavares (2019) and Tavares and Souza (2019), PN modeling has been chosen because it supports classical search algorithms through computational matrix representation, which have polynomial time complexity order (Russell, Norvig and Davis, 2010) and is adequate for full state representation in small scale problems, representing states, change mechanics and model mapping. In these cases the optimal path can be found using a simple BFS (Breadth-First Search), that expands PN places following a FIFO (First In First Out) list until the desired final state is found. The mapping between layers is the communication between PNRD, iPNRD and Physical Petri Space that allows integration and actuation (Tavares and Souza, 2019). Other representation paradigms and search methods are better suited for bigger state spaces and methods, and this case is not dealt in this paper.

Murray, Li and Satri (1994) introduces inverse kinematics for acting in pre-established spatial coordinates, in a constrained pick-and-place fashion. Guerin et al. (2018) applies reinforcement learning, multisensory and training datasets for robotic sorting. The method used in this work is not as resource consuming and complex as reinforcement learning and it is more versatile than pick-and-place because it contains a higher-level instruction set.

The motion planner used in this paper is a simple direct place drive directed by the Petri Space and operated by the motion driver in the microcontroller. Because of the discrete positions and orientation constraint make it so that more sensors and more complex dynamic instructions are not necessary. So inverse kinematics method could be applied to determine the exact actuation places (Murray, $\mathrm{Li}$ and Satri, 1994). To make it logically avoid a collision another line atop the original configuration is added so that the robot may move superior to the blocks when changing columns, making a collision impossible without external intervention.

In this approach places represents block state, robot state and position, and transitions identify actuation, dynamics and motion (Souza and Tavares, 2019). For each object (active or passive agent) there is only one marking at any time and it exhibits the current state in the specified Petri Model context. The discrete positions mapping are shown in Figure 2 and the corresponded Petri Space is presented in Figure 3. Both figures are complementary.

Solutions previously built upon the chosen modeling tools, 


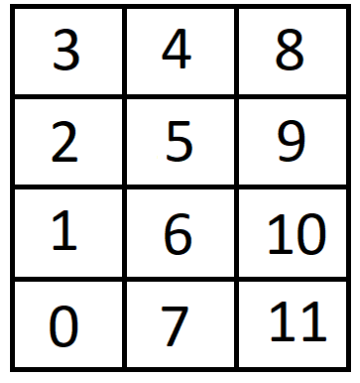

Fig. 2. Block World Discrete Position.

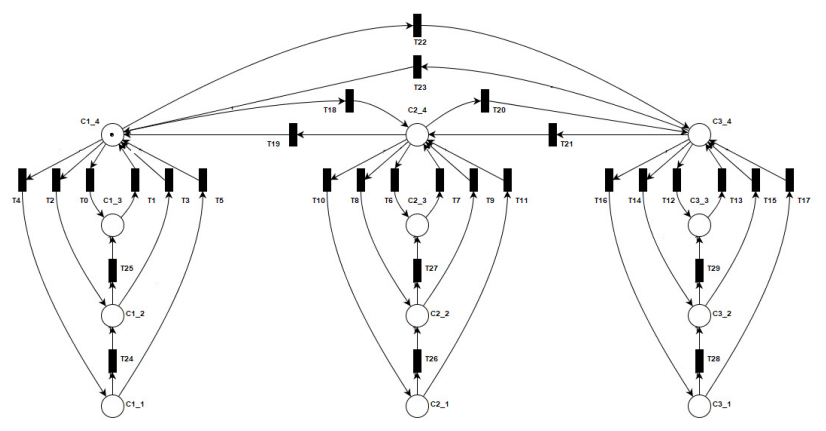

Fig. 3. Petri Space of Block World Domain with 3 Blocks.

definitions and constraints are presented in the next subsection with PNRD and iPNRD model. After it, the adaptive control is detailed.

\subsection{PNRD and iPNRD Model for Block Worlds}

This proposed modeling is based on three distinct levels. One models the passive agent environmental perspective, the Block PNRD. The second models the active agent global perspective, the robotic arm iPNRD. The third model the blocks positions and allowed movements, it means the integration of PNRD, iPNRD, and Petri State. These models communicate through tables (Souza and Tavares, 2019). The system works by passive agent state data gathering, the active agent generates a plan and actuates on the physical layer.

The passive agent model has only one relevant characteristic, it means to identify what entity is beneath the block. This generates 3 possible states and 6 transitions, as exemplified in Figure 4, regarding block A. Other blocks have a similar PNRD model.

When the robotic arm performs the identification of the block, it must inform its own id, its current PNRD state and the desired one.

The iPNRD represents the active agent perspective on the problem, which is also viewed as the global system state for this specific problem. The resulting PN has 13 logical states and 30 transitions (Figure 5). The transitions represent the change in position of a block but do not have concerns for the kinematic in movement. As this iPNRD model represent all system states, it is possible to generate a plan in order to navigate from an initial state to the desired one, identifying the necessary intermediary states and transitions by searching. This plan is a iPNRD transition firing sequence.

On the Petri State (Figure 3) the block movement takes

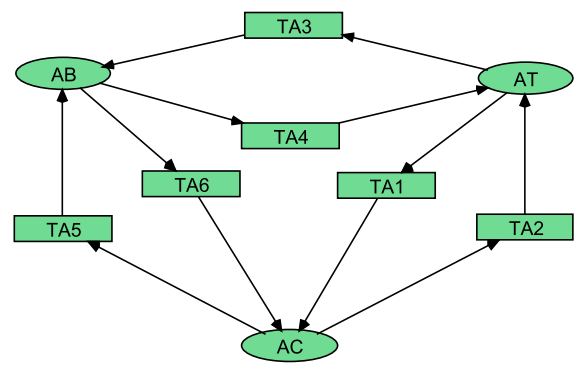

Fig. 4. Block A PNRD model (Tavares and Souza, 2019).

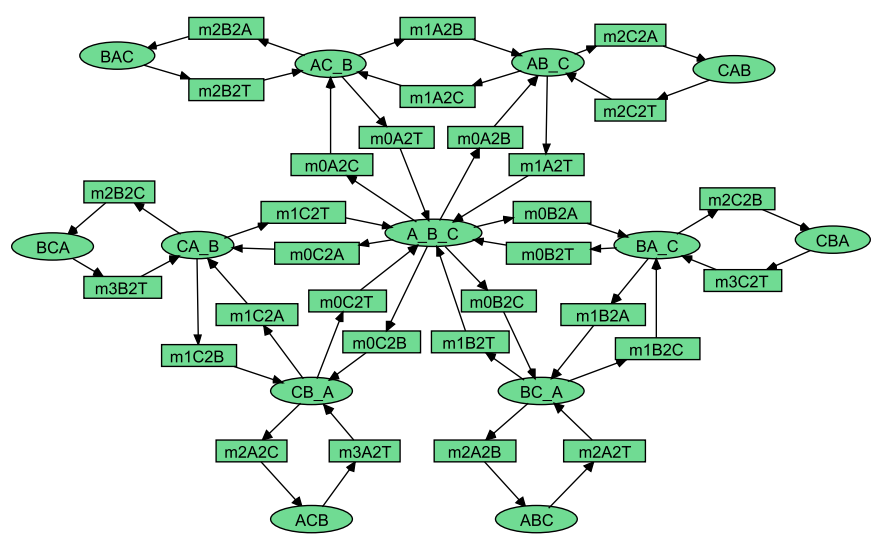

Fig. 5. Robotic Arm iPNRD model (Tavares and Souza, 2019).

place. This model has 12 positions, as it is a $4 \times 3$ discrete grid, and 22 transitions, initially. Its transitions relate directly to physical displacement. Each iPNRD transition refers to a sub objective Petri Space with a defined initial and final state, so a kinematic planning can also be performed by another BFS searching. The Petri Space plan is then processed to be fed to the robotic arm drive engine. The models integration follows a requisition, planning and actuation method. First the desired final state of each block $k_{i d}$ must be obtained, as well as its initial position. Then, using a conversion table, the three Block PNRD and three initial positions (perceived directly by the robotic arm) are converted into iPNRD objective and original states.

Afterwards a search in iPNRD model is performed to obtain the required macro transitions to reach the desirable state from the initial one. The next step is to obtain the necessary movements to execute the transitions. Finally actuation is processed once the robot driver acquires the movement string. A robotic arm movement request only can be performed if it is found by the PNRD, iPNRD and Petri State conversion tables, otherwhise an exception must be fired. This macro process is defined as the adaptive control sorting engine presented in the next subsection.

\subsection{Adaptative Control Sorting Engine}

In order to realize the necessary step of the Block World solution, an initial identification sweep is required. The blocks must be located in the Petri Space and the information inside each block must be aquired. Based on these informations the iPNRD states (initial and final, respec- 


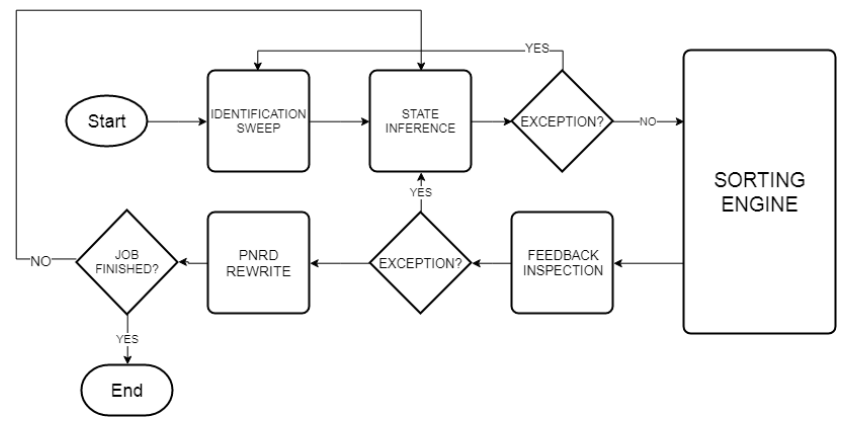

Fig. 6. Adaptive Control Sorting Engine (Souza and Tavares, 2019).

tively) are inferred, the sorting engine may be fed with the original and desired states. An initial exception can be raised if the final state from all blocks are not coherent, or if the initial state does not correspond with the physical positioning. The identification process is performed by reading all positions starting at the first column lowest position (Position 0 from Figure 2) and moving upwards until the third line. The same is repeated for columns two (positions 7, 6 and 5) and three (positions 11, 10 and 9). If the state inference does not raise an exception, the sorting engine starts. After the finishing of the sorting engine, a feedback inspection is added to guarantee that the objective was correctly performed. As the system is partially observable, the sorting engine may fail if an external agent acts without robotic arm perception or even due to robot malfunction. So a feedback inspection, which follows the same rules as the identification sweep, is performed. If an exception is raised, another state inference has to be performed in order to reorder the blocks. If no exception raises, the last step is to rewrite all blocks PNRD and to query for more movements until reach the final or desirable state. This adaptive control sorting engine is presented in Figure 6 .

Collendanchise and Ogren (2018) stated PN provide an alternate to FSM (Finite State Machines) that support concurrent systems and BT (Behavior Trees) supporting a modular design. The process flowchart described in Figure 6 consists of macro processes modeled by PN that contain their inner logic and internal micro processes, being similar to a BT in its modular approach. The modular representation is very useful for high level programming and understanding in very specialized activities, such as robot programming and agent design, NPC (Non Played Characters) in videogames as an example.

The adaptive controller acts on demand and does not require human intervention to operate. The system handling capacities is limited by the models and hardware, so improvements and adaptations might make it more robust and faster. The next section presents how it is possible to generate an automatic column assigment and, consequently, to optimize the identification sweep and the feedback inspection.

\section{AUTOMATIC COLUMN ASSIGMENT AND MOVEMENT OPTIMIZATION}

The sorter previously described in the subsection 3.2 had a placing restriction that did not allow for more than 13 physical configurations so that each logical configuration

\begin{tabular}{|c|c|c|}
\hline \multicolumn{3}{|c|}{ Reference - 0 } \\
\hline 3 & 4 & 8 \\
\hline 2 & 5 & 9 \\
\hline 1 & 6 & 10 \\
\hline 0 & 7 & 11 \\
\hline$X$ & $Y$ & $Z$ \\
\hline
\end{tabular}

\begin{tabular}{|c|c|c|}
\hline \multicolumn{3}{|c|}{ Permutation - 1 } \\
\hline 3 & 8 & 4 \\
\hline 2 & 9 & 5 \\
\hline 1 & 10 & 6 \\
\hline 0 & 11 & 7 \\
\hline$X$ & $Z$ & $Y$ \\
\hline
\end{tabular}

\begin{tabular}{|c|c|c|}
\hline \multicolumn{3}{|c|}{ Permutation - 2 } \\
\hline 4 & 3 & 8 \\
\hline 5 & 2 & 9 \\
\hline 6 & 1 & 10 \\
\hline 7 & 0 & 11 \\
\hline $\mathrm{Y}$ & $\mathrm{X}$ & $\mathrm{Z}$ \\
\hline
\end{tabular}

\begin{tabular}{|c|c|c|}
\hline \multicolumn{3}{|c|}{ Permutation - 3 } \\
\hline 4 & 8 & 3 \\
\hline 5 & 9 & 2 \\
\hline 6 & 10 & 1 \\
\hline 7 & 11 & 0 \\
\hline$Y$ & $Z$ & $X$ \\
\hline
\end{tabular}

\begin{tabular}{|c|c|c|}
\hline \multicolumn{3}{|c|}{ Permutation - 4 } \\
\hline 8 & 3 & 4 \\
\hline 9 & 2 & 5 \\
\hline 10 & 1 & 6 \\
\hline 11 & 0 & 7 \\
\hline$Z$ & $X$ & $Y$ \\
\hline
\end{tabular}

\begin{tabular}{|c|c|c|}
\hline \multicolumn{3}{|c|}{ Permutation - 5 } \\
\hline 8 & 4 & 3 \\
\hline 9 & 5 & 2 \\
\hline 10 & 6 & 1 \\
\hline 11 & 7 & 0 \\
\hline$Z$ & $Y$ & $X$ \\
\hline
\end{tabular}

Fig. 7. Configuration types of Dynamic Column Assignement.

had one and only one corresponding material configuration. In this section an adaptable method that allows for multiple physical correspondents is explored.

As stated in Hofer (2017), autonomous robots have to adapt their decisions to their environmental circumstances. A physical configuration which is not modeled in the previous inference table might be possible, requeries that the robot must be instructed to deal with all possibles physical compositions. This is performed by expanding the model to deal with these new configurations. One way to solve this problem is to expand the State Space transitions to match the total amount of place movement in the physical configurations, but this solution generates a state explosion depending on column numbers and the quantity of blocks.

Another approach does not change the logical iPNRD and Petri Space size, mantaining the sorting engine logic. This approach is the dynamic column attribution that is used to make the system work with all 60 physical configurations but searching in a 13 sized logical Petri Space, at the cost of having an expanded inference table with an extra output parameter, the configuration type. The configuration type table must contain all accepted physical dispositions.

The robot has partial observation of the Petri Space at all times, due this system has only RFID reader in the robotic arm as sensor. It does not know, before the identification process, what configuration type the blocks are in. The configuration type is determined by the blocks physical disposition, which is also inferred in the state inference. The system now must feed the sorting engine three parameters: original and objective states and configuration type. The configuration types are 6 and it is represented in Figure 7, where Reference 0 is the standard configuration. Each letter (X, Y or Z) indexes a column, for simplification. The difference between each configuration type is the column arrangement. Because there are 3 distinct columns they can be permuted in 3 ! ways, giving a total of 6 . In 7 of the $13 \mathrm{iPNRD}$ states there are 6 corresponding physical permutations for each. The remainder 6 have only 3 each. As example imagine ABC. This set can be put on only three distinct columns. The same applies for the other 5 
full stacked states, having $3 ! / 2$ permutations each.

The problem that arises from the full stacked states is that 2 configuration types may satisfy the requisites. In order to solve this problem a guideline is necessary, being the total cost of the possible configurations, the optimal one being picked.

Inside the sorting engine, the $\mathrm{iPNRD}$ and PNRD remains exactly the same, but there will be changes to the Petri Space and drive engine. This is the reason to why the configuration type is an input parameter. The logical solution remains the same, the Petri Space is mildly altered and the drive engine is highly dependent of the configuration type.

The changes in the Petri Space happen on the column transitions. Imagine the reference configuration $X_{-} Y_{-} Z$. The column transitions indicate a change in the physical position of the claw, going from $\mathrm{X}$ to $\mathrm{Y}$, or $\mathrm{Y}$ to Z. Now, in the configuration $Z_{-} X_{-} Y$ the $\mathrm{X}$ to $\mathrm{Y}$ and $\mathrm{Y}$ to $\mathrm{Z}$ transitions would not mean the same position exchange. To solve this the Petri Space has to be mutated in the two column transition pairs in order for the $\mathrm{PN}$ to reflect the actual position shift.

The Adaptive Control Sorting Engine is highly sensible towards the configuration type. For each material position there is a drive sequence that makes the robot claw reach the discrete position. The commands are directly related to the material configuration, not to the Petri Space. Imagine the reference configuration and $X_{-} Z_{-} Y$. The drive commands in the $X$ column are exactly the same in both, but that does not happen in the $\mathrm{Y}$ and $\mathrm{Z}$ columns, but the command to get to the reference material positions 6 or 10 remain the same. This means that the configuration type is a parameter that is necessary for interpreting the drive sequence, altering the driving table accordingly to the permutation type. Dynamic Column attribution does not increase time costs but will increase space costs because it is required for all physical configurations to be modeled in the inference table. There will be variation in the Petri Space column changing transitions and the drive interpreter according to the permutation type, requiring additional data to be stored and the configuration type to be an input parameter.

\subsection{Movement Optimization}

The identification and feedback processes described in section 3.2 are logically fail proof but lose in operational speed when compared to optimized methods. In this section the identification and feedback dynamics are improved through the use of an expanded model and known environmental conditions and restrictions (Fakour, Kosari and Jafarzadeh, 2018) to formulate a behavior plan.

The improved identification sweep takes into consideration logical restrictions and model parameters. The system works as represented in Figure 8. Initially, in the setup, the robot moves from a reference point towards the first discrete position, being the lowest point of the first column $(\mathrm{Col}=0$, Line $=0)$. The next step is to attempt a tag read. The RFID reader gets tag data and positioning. It infers if any exception occurs, it means, if the initial positioning is coherent with the tag state and if the total tags final state are consistent. If an exception raises, it must be treated. In case of divergence of initial positioning with tag state,

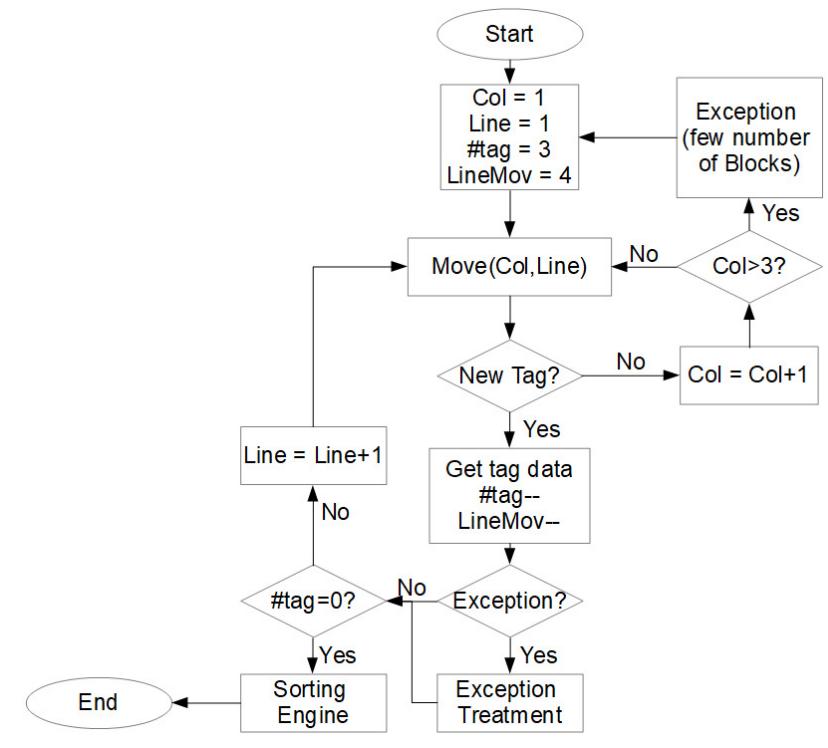

Fig. 8. Optimized Identification Sweep.

a new writing must update the tag state following the positioning system.

After exception treatment or in case of no exception, the robot must verify if all tags were perceived. If not, it goes to the next line. If it is the last line the robot must move to the next column. In case the last column was reached, it means that fewer blocks were read and the robot must restart the sweep. When all data tags were captured the sorting engine can be performed.

In case the identification is successful the acquired data is fed into the inference table, to generate initial and objective states for the sorting engine. If the objective state is unobtainable the system leaves a warning and ends the iteration.

This system optimizes the number of robot movement based on the total amount of tags which, in this specific implementation, is fixed in three. As an example imagine the configuration $A_{-} C B$. Because tag $A$ is identified successfully first and there cannot be more than three tags in the system the robot claw does not need to move from one column to another in the forth line (LineMov parameter). In this example, after the detection of block $A$, the robot knows it can change columns in the third line without collision risk. So this expands the model by adding more possible transitions in Petri State allowing movements in other columns as new tags are identified.

Another improvement aspect is the total amount of tag read query performed. In the simple identification process the robot always read all 9 possible discrete positions that the blocks could occupy. In the improved version the tag read query is performed 3 times in the best case and 5 times in the worst cases because in case of no tag reading the robot changes the column immediately. Another exception arises if the number of tag readings was less than 3 and the sweep restart.

The improved feedback process works by searching for the closest not inspected block, in its expected position. The process continues until all tags are correctly identified in their desired states or an exception arises. In case the process is successful the PNRD rewrite process takes place, where the robot will do the inverse transition sequence 
and update data in the tags. In case a tag is not identified or is in the wrong position, a warning occurs and ends the iteration. Because of the way this new process is constructed another identification sweep might be required if the tags are not all found.

This procedure also expands the model. More transitions are allowed because of the knowledge on the expected position of each block. The system must take the optimal route that does not result in a collision.

The sorting process speed is improved by the two optimized processes. Because robot movement is the slowest activity in the system, adding complexity to the models and computational processes makes the sorting process faster as less dynamic comands are required.

\section{CONCLUSION}

This paper presented an automatic column assigment for Block World domain with PNRD/iPNRD. This solution allows any kind of physical arrangement of three blocks to be perceived and moved until the desired final positioning. Although this paper deals with a full system model, for more complex domains, this solution is impractical, requiring integration of the $\mathrm{iPNRD}$ model with Automatic Planner result to check if a specific plan was followed. PNRD and iPNRD exceptions can assist online replanning. Another improvement is that Petri State transition could be expanded enabling new transitions depending on the certainty that no collisions will occur in a given case. There is also the dynamical (movement) and geometrical aspect. Regarding dynamics and concatenation of movement, there is much that can be done to minimize the amount of displacements. There are dynamical and geometrical issues regarding the blocks disposition and manipulation. In order to successfully realize the actuation and sensing the system must be precise or robust, meaning that motors torque, potency and control, material strength analysis, sensor signal studies and physical references to assure the fidelity of the driver system motion might be required.

In this work we used absolute geometrical references for block and robot placement and attitude. There can be many different types of exceptions through the process of solving the system, assuming every model and table was created correctly. Exceptions in the system identification might mean that a block is missing or an RFID element is malfunctioning. In the state generator it means that the desired final state doesn't exist in the model because there is an interest crisis within the PNRD that causes a confrontation. Or the blocks require a physically impossible configuration. The exception identification generates the adaptive event control, meaning that if any failure or external actuation is identified this approach is able to solve it automatically. As this control is in the RFID reader level, it is an Edge Computing solution.

Three blocks model is simple and easy to be verified without formal methods. However, for more complex systems a previous formal verification is demanded.

\section{ACKNOWLEDGMENTS}

\section{REFERENCES}

Colledanchise, M., Ogren, P. 2018. Behavior Trees in Robotics and AI. Robotics [cs.RO]. arXiv:1709.00084v3.

Fakoor, M., Kosari, A., Jafarzadeh, M. 2016. Humanoid robot path planning with fuzzy Markov decision processes. Journal of Applied Research and Technology. Vol. 14, Issue 5, pp. 300-310.

Fonseca, J.P.S., 2018. High-level Petri nets and inverted PNRD associated to mobile robot control: an approach to search and rescue on trails and crossings. Ph.D.thesis. Universidade Federal de Uberlândia, , Uberlândia, Brasil

Fonseca, J.P.S., de Sousa, A.R., Ferreira, M.V.M, Tavares, J.J.P.Z.S. (2016) PlanPAS: PLC and automated planning integration, International Journal of Computer Integrated Manufacturing, 29:11, 1200-1217, DOI: 10.1080/0951192X.2015.1067909

Guérim, J., Thiery, S., Nyiri, E., Gibaru, O., 2018. Unsupervised Robotic Sorting: Towards Autonomous Decision Making Robots. International Journal of Artificial Intelligence and Applications, v. 9, n. 2, pp. 81-98.

Ghallab, M., Nau, D.S and Traverso, P., 2014. The Actors View of Automated Planning and Acting: A Position Paper. Artificial Intelligence 208: 1-17. doi:10.1016/j.artint.2013.11.002.

Gupta, N., Nau, D.S., 1992. On the complexity of blocksworld planning. Artificial Intelligence. 56, pp 223-254.

Hofer, L. 2017. Decision Making algorithms for autonomous robots. Robotics [cs.RO]. Université de Bordeaux, 2017. English.

Murata, T., 1989. Petri Nets: "Properties, analysis and applications". Proceedings of the IEEE, v. 77, n.4, p. 541-580.

Murray, R. M., Li, Z., Sastry, S. S., 1994. A Mathematical Introduction to Robotic Manipulation. CRC Press, 1st ed.

Russell, S.J., Norvig, P., Davis, E., 2010. Artificial Intelligence: A Modern Approach. Prentice Hall, New Jersey, 3rd ed.

Sette, F. M., T. S. Vaquero, S. W. Park, and J. R. Silva. 2008. "Are Automated Planners up to Solve Real Problems?" In Proceedings of the 17th IFAC World Congress, edited by M. J. Chung and P. Misra, Seoul, July 6-11. International Federation of Automatic Control. ISBN 978-3-902661-00-5.

Souza, G.A., Tavares, J.J.P.Z.S., 2019. Adaptive Discrete Event Control Based on Integration of PNRD and iPNRD Applied to Block World Domain. To be published in 25th International Congress of Mechanical Engineering - COBEM 2019, Uberlândia, Brazil.

Tavares,J.J.P.Z.S., Saraiva,T.A., 2010. "Elementary Petri net inside RFID distributed database (PNRD)". International Journal of Production Research Vol. 48, Issue 9, pp. 25632582.

Tavares, J.J.P.Z.S., Souza, G.A., 2019. PNRD and iPNRD Integration Assisting Adaptive Control in Block World Domain. In ONSE 2019 International Workshop on Petri Nets and Software Engineering, 2019, Aachen. CEUR Workshop Proceedings, 2019, v.2424, p.73-90. Available at http://ceur-ws.org/Vol-2424/paper6. pdf 